\title{
Fatores de Risco Associados à Recusa de Notas de Transferência e Vales Cirurgia: O Caso da Região Centro em Portugal
}

\section{Risk Factors Associated with the Refusal of Surgery Vouchers: The Case of Central Portugal}

\author{
Salomé CRUZ1 ${ }^{1}$, Carlota QUINTAL $\square^{2,3}$, Patrícia ANTUNES 3,4 \\ Acta Med Port 2022 Mar;35(3):201-208 - https://doi.org/10.20344/amp.15357
}

\section{RESUMO}

Introdução: Em Portugal, a recusa de Notas de Transferência e Vales Cirurgia é elevada, dificultando o cumprimento dos tempos máximos de resposta garantidos para cirurgias eletivas. Os objetivos deste estudo foram analisar a evolução de notas e vales emitidos/ recusados para o período compreendido entre o terceiro trimestre de 2016 e o quarto trimestre de 2019 e os fatores de risco associados à sua recusa, na Região Centro, em Portugal.

Material e Métodos: Os dados provêm da base de dados de notas/vales cancelados e da lista de inscritos para cirurgia a 31 de dezembro de 2019. $\mathrm{Na}$ análise dos fatores de risco recorremos à regressão logística múltipla.

Resultados: A emissão de notas/vales aumentou após 2018 e as taxas de recusa de transferência mantiveram-se acima dos $55 \%$ a partir do terceiro trimestre de 2018. A chance de recusa foi maior para idades superiores a 55 anos $(O R=1,136 ;$ IC $=1,041-1,240$; $\mathrm{OR}=1,095 ; \mathrm{IC}=1,005-1,194 ; \mathrm{OR}=1,098 ; \mathrm{IC}=1,002-1,203$, para as faixas etárias $55-64,65-74$ e $75-84$, respetivamente) para a cirurgia convencional, quando comparada com ambulatório $(\mathrm{OR}=2,498$; IC $=2,343-2,663)$ e para a especialidade de Ortopedia, quando comparada com Cirurgia Geral $(O R=1,123$; IC $=1,037-1,217)$. A chance de recusa variou também entre hospitais (por exemplo $\mathrm{OR}=3,853$; IC = 3,610 - 4,113; OR = 3,600; IC = 3,171 - 4,087; OR = 2,751; IC = 3,383-3,175 e OR = 1,337; IC= 1,092 1,637, para os hospitais de origem identificados como HO_2, HO_7, HO_4 e HO_6, respetivamente).

Conclusão: Neste estudo confirmou-se que a emissão de notas de transferência/vales cirurgia aumentou após a redução legal dos tempos máximos de resposta garantidos em 2018 e que as taxas de recusa de transferência vinham já a registar uma tendência de aumento desde 2016 , tendo-se mantido acima dos $55 \%$ a partir do terceiro trimestre de 2018 . Alguns fatores para os quais se encontrou uma associação positiva com a recusa são a idade, a cirurgia convencional (em comparação com ambulatório) e a especialidade de Ortopedia (em comparação com Cirurgia Geral).

Palavras-chave: Acesso aos Serviços de Saúde; Fatores de Risco; Listas de Espera; Portugal; Procedimentos Cirúrgicos; Procedimentos Cirúrgicos Eletivos

\section{ABSTRACT}

Introduction: In Portugal, the rate of refusals regarding transfer between hospitals through surgery vouchers is high, which makes it difficult to meet maximum waiting times for elective surgeries. The objectives of this study are to examine how many vouchers were issued and refused between the third quarter of 2016 and the fourth quarter of 2019 and the risk factors associated with their refusal, in Central Portugal

Material and Methods: Data was obtained in the database of cancelled vouchers and the waiting list for surgery on the $31^{\text {st }}$ December 2019. Multiple logistic regression was used to investigate risk factors.

Results: The number of issued vouchers increased after 2018 and the rate of refusals has been above $55 \%$ since the $3^{\text {rd }}$ quarter of 2018. Refusal was more likely for individuals aged 55 years or above $(\mathrm{OR}=1.136 ; \mathrm{Cl}=1.041-1.240 ; \mathrm{OR}=1.095 ; \mathrm{Cl}=1.005-1.194$; $\mathrm{OR}=1.098 ; \mathrm{Cl}=1.002-1.203$, for the age bands $55-64,65-74$ and $75-84$, respectively), for inpatient surgery when compared to ambulatory $(\mathrm{OR}=2.498 ; \mathrm{Cl}=2.343-2.663)$ and for Orthopaedics when compared to General Surgery $(\mathrm{OR}=1.123 ; \mathrm{Cl}=1.037-$ 1.217). The odds of refusal also varied across hospitals (for example $\mathrm{OR}=3.853 ; \mathrm{Cl}=3.610-4.113 ; \mathrm{OR}=3.600 ; \mathrm{Cl}=3.171-4.087$; $\mathrm{OR}=2.751 ; \mathrm{Cl}=3.383-3.175$ e OR $=1.337 ; \mathrm{Cl}=1.092-1.637$, for hospitals identified as HO_2, HO_7, HO_4 and HO_6, respectively).

Conclusion: In this study, we have confirmed that the number of issued surgery vouchers increased after the administrative reduction of maximum waiting times in 2018 and that the rate of transfer refusals has been increasing since 2016 and has remained above $55 \%$ from the third trimester of 2018 onwards. Some of the factors for which we obtained a positive association with refusal are age, inpatient surgery (compared to ambulatory) and Orthopaedics (compared to General Surgery).

Keywords: Elective Surgical Procedures; Health Services Accessibility; Portugal; Risk Factors; Surgical Procedures; Waiting Lists

\section{INTRODUÇÃO}

As listas e os tempos de espera para cuidados de saúde em geral, e para cirurgias eletivas em particular, constituem uma importante questão da política de saúde na maioria dos países da Organização para a Cooperação e Desenvolvimento Económico (OCDE). ${ }^{1}$ Em alguns países da OCDE os tempos de espera para cirurgias eletivas estagnaram na última década mas em outros começaram a subir mesmo antes da pandemia por COVID-19. ${ }^{2}$ Embora possam existir vários constrangimentos e tempos de espera nos diversos contactos do utente com os serviços de

1. Economia Financeira. Faculdade de Economia. Universidade de Coimbra. Coimbra. Portugal.

2. Centre for Business and Economics Research (CeBER). Faculdade de Economia. Universidade de Coimbra. Coimbra. Portugal.

3. Centro de Estudos e Investigação em Saúde da Universidade de Coimbra (CEISUC). Universidade de Coimbra. Coimbra. Portugal.

4. Unidade Regional de Gestão do Acesso. Administração Regional de Saúde do Centro. Coimbra. Portugal.

$\square$ Autor correspondente: Carlota Quintal. qcarlota@fe.uc.pt

Recebido: 19 de novembro de 2020 - Aceite: 17 de maio de 2021 - First published: 04 de janeiro de 2022 - Online issue published: 02 de março de 2022 Copyright ( ) Ordem dos Médicos 2022 
saúde, a interpretação mais comum de tempo de espera para cirurgia eletiva corresponde ao tempo que medeia a data em que o utente é adicionado à lista de espera, para um determinado procedimento e após a avaliação de um especialista, e a data em que é admitido num serviço para a realização do procedimento em causa. ${ }^{3}$ Esta é também a interpretação subjacente ao presente trabalho.

Os tempos de espera ocorrem quando há um desequilíbrio entre a oferta e a procura de serviços de saúde. Num cenário de mercado tradicional, o preço ajusta-se promovendo o encontro entre a oferta e a procura. No setor da saúde e em países onde existe cobertura universal, combinada com copagamentos nulos ou diminutos e recursos limitados, os tempos de espera emergem como um mecanismo de racionamento alternativo ao preço e independente da capacidade de pagar. ${ }^{4}$ Tendências como o aumento da esperança de vida da população, do número de cidadãos com doença crónica e do aparecimento de novos medicamentos e tecnologias em saúde contribuem para aumentar as listas de espera, enquanto o aumento da oferta de serviços e da produtividade dos prestadores concorrem para a redução dessas listas. ${ }^{1}$

Do ponto de vista da eficiência, os tempos de espera são relevantes para evitar a subutilização da capacidade instalada ${ }^{5}$ e podem mesmo pressionar os hospitais a aumentarem a sua produtividade. ${ }^{1,3}$ No entanto, longos tempos de espera podem acarretar aumento de custos para os prestadores devido à gestão das listas de espera. ${ }^{6}$ Da perspetiva dos utentes são várias as desvantagens associadas aos tempos de espera como sejam a deterioração do seu estado de saúde e eventualmente da sua autonomia, prolongamento do sofrimento e a ansiedade, todos confluindo na redução da qualidade de vida. ${ }^{3,7-9}$ Adicionalmente, existe evidência ao nível dos países da OCDE de tempos de resposta desiguais para cuidados prestados mesmo no setor público. Esta desigualdade tende a ser favorável aos indivíduos com estatuto socioeconómico mais elevado..$^{10,11}$

Em face destes problemas, têm sido adotadas algumas medidas de política para combater tempos de espera considerados excessivos. Algumas são direcionadas para a oferta como o aumento temporário dos serviços oferecidos com financiamento extra, aperfeiçoamento da gestão das listas, subcontratação aos setores privado e social ou pagamentos baseados na atividade por forma a incentivar maior eficiência. Do lado da procura existem exemplos, tais como normas para priorizar doentes e subsidiação de seguros privados. Pela sua maior eficácia reconhecida, temos assistido nos países da OCDE a um enfoque crescente em políticas combinadas como por exemplo os tempos máximos de resposta garantidos (TMRG), associados ou não a sanções, e à escolha dos utentes e concorrência entre prestadores. ${ }^{1,12} \mathrm{O}$ objetivo dos TMRG é assegurar a prestação do serviço requerido dentro de um período de tempo, previamente definido de acordo com o que é considerado clinicamente aceitável em cada caso tipo.

Em muitos países, os TMRG têm sido cada vez mais associados à escolha de prestador por parte dos utentes como forma de impor efetivamente os tempos máximos que se pretendem garantir. ${ }^{1} \mathrm{Em}$ teoria, espera-se que a possibilidade de escolha conduza ao aumento da qualidade dos serviços prestados e da produtividade devido à concorrência entre instituições no intuito de atrair utentes e consequentemente o financiamento respetivo. ${ }^{13}$ Por sua vez, a qualidade dos cuidados percebida pelos utentes dependerá de diversas determinantes como por exemplo a reputação do hospital, a competência dos profissionais e o relacionamento interpessoal. ${ }^{14,15}$ Não obstante a influência previsível da qualidade dos serviços sobre a escolha dos utentes, este processo é complexo, envolvendo outros fatores. Desde logo a escolha fica sujeita à condição do procedimento ser oferecido pela instituição pretendida. Adicionalmente, a escolha pode ser influenciada pela recomendação de conhecidos ou de profissionais de saúde bem como pela localização, existindo uma preferência por instituições próximas da área de residência sobretudo no caso de utentes idosos. ${ }^{16}$

Em Portugal, os tempos de espera têm também constituído motivo de preocupação no âmbito da política de saúde ao longo das últimas décadas. Deste modo, foram sendo adotadas medidas, destinadas, não só mas essencialmente, ao combate às listas de espera cirúrgicas. O programa mais antigo, o Programa Específico de Recuperação de Listas de Espera, teve uma duração de três anos entre 1995 e 1998; outros Ihe seguiram. ${ }^{1,17}$ Em 2004, foi implementado o Sistema Integrado de Gestão de Inscritos para Cirurgia (SIGIC), ainda em vigor, e que tem por objetivo gerir, de forma integrada e continuada, a lista de inscritos para cirurgia nos estabelecimentos do Serviço Nacional de Saúde (SNS). A Lei n. ${ }^{\circ} 41 / 2007$ de 24 de agosto ${ }^{18}$ veio introduzir o conceito de TMRG no quadro da Carta dos Direitos de Acesso aos Cuidados de Saúde no SNS, sendo os tempos específicos definidos mais tarde em 2008 pela Portaria n. ${ }^{0} 1529 / 2008$ de 26 de dezembro. ${ }^{19}$ Também em 2008, foi instituída a liberdade de escolha do utente, pela Portaria n. ${ }^{\circ}$ 45/2008 de 15 de janeiro. ${ }^{20}$ De acordo com este modelo, em Portugal, quando 50\% do TMRG é atingido é emitida uma Nota de Transferência (NT) que permite ao utente realizar a sua cirurgia noutro hospital público com disponibilidade. Aos $75 \%$ do tempo é emitido um Vale Cirurgia (VC) que permite ao utente realizar a sua cirurgia num conjunto mais alargado de instituições do setor público, privado e social. Os utentes têm o direito de recusar a transferência do processo, tendo a circunstância de um elevado número de recusas sido identificada há mais de uma década. ${ }^{21}$ No triénio 2014 - 2016 a taxa de utilização de NT/VC continuava muito baixa, situando-se nos $20 \%{ }^{22}$ A Portaria n. ${ }^{\circ} 153 / 2017$ de 4 de maio, ${ }^{23}$ com efeitos a partir de 1 de janeiro de 2018, veio implementar a redução dos TMRG, o que significa que os utentes passam a receber as NT/VC mais cedo, o que pode aumentar ainda mais a já elevada recusa destes mecanismos.

Neste contexto, torna-se pertinente perceber quais os fatores de risco associados à recusa de NT/VC com vista a delinear estratégias que aumentem a sua eficácia, 
contribuindo desse modo para um melhor cumprimento dos TMRG.

Tanto quanto é do nosso conhecimento, o único estudo sobre recusa de transferência usa dados de 2007,21 baseando-se numa metodologia e amostra diferentes das que são utilizadas neste trabalho.

Os objetivos deste estudo são assim investigar esses fatores de risco para o caso da Região Centro e analisar a evolução de NT e VC emitidos e recusados entre o terceiro trimestre de 2016 e o quarto trimestre de 2019.

\section{MATERIAL E MÉTODOS}

Para o presente estudo foram recolhidos dados relativos à Região Centro e referentes a: i) número de NT/VC emitidos mensalmente, extraídos pela Administração Central do Sistema de Saúde (ACSS, IP), para o período entre 1 de julho de 2016 e 31 de dezembro de 2019; ii) NT/VC cancelados, por motivo, extraídos do Sistema Integrado de Gestão da Lista de Inscritos para Cirurgia (SIGLIC), para o período entre 1 de julho de 2016 e 31 de dezembro de 2019; iii) lista de inscritos para cirurgia (LIC) em 31 de dezembro de 2019, extraída do SIGLIC. A extração da informação do SIGLIC foi efetuada pela Administração Regional de Saúde do Centro (ARSC, IP).

A base de dados de NT/VC cancelados continha inicialmente 87215 observações com informação acerca do hospital de origem $(\mathrm{HO})$, especialidade cirúrgica no $\mathrm{HO}$, número da posição que ocupa na LIC do $\mathrm{HO}$, motivo de cancelamento e data de cancelamento do vale. Para analisar a evolução trimestral de NT/VC emitidos e cancelados, apenas se considerou o motivo 'Recusa de Transferência', sendo neste caso a amostra constituída por 71504 observações.

A base de dados da LIC continha inicialmente 50382 episódios. Cada episódio compreende informação respeitante a todo o processo clínico do utente: número do processo de origem, número do processo de destino, data de inclusão, prioridade, idade do utente, sexo, $\mathrm{HO}$, número da LIC de origem, número da LIC de destino, especialidade cirúrgica, código de intervenção e sua respetiva designação, modalidade de cirurgia (convencional ou ambulatório), código da patologia, patologia, tempo de espera (em meses), estado atual do utente, data de agendamento, localidade do utente, código do grupo de extração e sua respetiva descrição, código do grupo nosológico e sua respetiva descrição, e médico proponente.

$\mathrm{Na}$ análise dos fatores de risco associados à recusa de NT/VC, este estudo apenas considera os utentes maiores de idade, no pressuposto de não serem os menores a decidir sobre a recusa, ou não, de NT/VC. Por conseguinte, foram eliminadas 3291 observações da base de dados inicial.

Face aos novos valores dos TMRG definidos para cada patologia e nível de prioridade, e perante o tempo de espera e nível de prioridade para cada episódio clínico indicado na LIC, determinou-se em que estádio de tempo de espera se encontrava cada episódio: inferior a $50 \%$, igual ou superior a $50 \%$ e inferior a $75 \%$, igual ou superior a $75 \%$ e inferior a $100 \%$, ou superior a $100 \%$ do TMRG. Para a análise dos fatores de risco associados à recusa, assumiu-se que todos os episódios com tempos de espera inferiores a $50 \%$ não se qualificavam para NT ou VC pelo que foram eliminados da base de dados. Deste modo, a amostra final utilizada na análise de regressão corresponde a 33153 observações.

Nesta análise, considerou-se como variável dependente a variável binária 'Recusa' que assume o valor 1 se o utente recusou NT/VC e 0 , caso contrário. Para construir esta variável, comparou-se o número da LIC de origem presente na base de dados de NT/VC cancelados com o número da LIC de origem presente na base de dados dos episódios que aguardavam resolução cirúrgica em 31 de dezembro de 2019. Quando os números das LIC coincidem, significa que houve cancelamento de NT/VC por recusa de transferência. Assim, nos casos em que os números das LIC coincidem, a variável 'Recusa' toma o valor um, e nos casos em que o número da LIC dos episódios em espera em 31 de dezembro não tem correspondência na base de dados dos NT/VC cancelados a variável 'Recusa' toma o valor zero.

Como variáveis explicativas e tendo em conta a informação disponível na base de dados, selecionaram-se variáveis sociodemográficas e variáveis relativas ao processo clínico do utente. Para o efeito, consideraram-se o sexo e a idade dos utentes, o $\mathrm{HO}$, o nível de prioridade, a modalidade, a especialidade da cirurgia e o período de inscrição na lista de espera (se antes ou após a redefinição dos TMRG). A Tabela 1 apresenta a categorização destas variáveis, a respetiva descrição e a sua representatividade na amostra.

Para analisar a associação entre os diversos fatores considerados neste estudo e a recusa de NT/VC, recorremos à regressão logística múltipla. As análises foram realizadas com o programa SPSS $26.0^{\circledR}$. Os resultados são relatados sob a forma de razão de chances (odds ratio), também designado de razão de possibilidades. A razão de chances resulta da razão da chance de exposição (neste caso, a recusa de NT/VC) num determinado grupo dividido pela chance de exposição noutro grupo, tomado como categoria de referência. Por sua vez, a chance é também uma razão: da probabilidade de sucesso (recusa de NT/VC) e da probabilidade de insucesso (não recusa de NT/VC). A chance é assim um conceito diferente da probabilidade. Considerando por exemplo a variável sexo (tomando como referência o sexo 'feminino'), se 80 em 100 indivíduos do sexo masculino recusarem NT/VC, então a sua probabilidade de recusa é 0,8 e a sua probabilidade de não recusa é 0,2 . A chance de recusa entre os homens será de 80 para 20 , ou seja, 4 (= $0,8 / 0,2)$. Se, no caso das mulheres, 75 em 100 recusarem NT/VC, então, a sua probabilidade de recusa é 0,75 e a sua probabilidade de não recusa é 0,25 . A chance de recusa entre as mulheres será de $3(=0,75 / 0,25)$. Deste exemplo resulta uma razão de chances de $1,33(=4 / 3)$. Conclui-se que a chance ou possibilidade de recusa de NT/VC entre os homens é 1,33 vezes superior à chance de recusa de NT/VC entre as mulheres. 
Tabela 1 - Variáveis usadas na análise de regressão

\begin{tabular}{|c|c|c|}
\hline Variável & $\begin{array}{c}\text { Número de } \\
\text { observações } \\
(n=33 \text { 153) }\end{array}$ & $\begin{array}{c}\text { Frequência } \\
\text { relativa } \\
(\%)\end{array}$ \\
\hline \multicolumn{3}{|c|}{ Variável dependente } \\
\hline Recusa & 10372 & 31,3 \\
\hline \multicolumn{3}{|c|}{ Variáveis explicativas } \\
\hline \multicolumn{3}{|l|}{ Sexo } \\
\hline Masculino & 14240 & 43,0 \\
\hline \multicolumn{3}{|l|}{ Idade } \\
\hline Idade_18_44§ & 5165 & 15,6 \\
\hline Idade_45_54 & 4163 & 12,6 \\
\hline Idade_55_64 & 6058 & 18,3 \\
\hline Idade_65_74 & 8267 & 24,9 \\
\hline Idade_75_84 & 7577 & 22,8 \\
\hline Idade_85+ & 1923 & 5,8 \\
\hline \multicolumn{3}{|l|}{ Hospital de origem } \\
\hline HO_1§ & * & * \\
\hline HO_2 & * & * \\
\hline HO_3 & * & * \\
\hline HO_4 & * & * \\
\hline HO_5 & * & * \\
\hline HO_6 & * & * \\
\hline HO_7 & * & * \\
\hline HO_8 & * & * \\
\hline HO_9 & * & * \\
\hline HO_outros & * & * \\
\hline \multicolumn{3}{|c|}{ Nível de prioridade da cirurgia } \\
\hline Normal§ & 30536 & 92,1 \\
\hline Prioritário_MP & 2617 & 7,9 \\
\hline \multicolumn{3}{|c|}{ Modalidade da cirurgia } \\
\hline Cir_ambul§ & 16371 & 49,4 \\
\hline Cir_convenc & 16782 & 50,6 \\
\hline \multicolumn{3}{|c|}{ Especialidade da cirurgia } \\
\hline Cir_geral ${ }^{\S}$ & 5586 & 16,8 \\
\hline Oftalmologia & 10938 & 33,0 \\
\hline Ortopedia & 7977 & 24,1 \\
\hline Outras_esp & 8652 & 26,1 \\
\hline \multicolumn{3}{|c|}{ Período posterior/anterior à redefinição dos TMRG } \\
\hline Ano_inclusão ${ }^{\dagger}$ & 31406 & 94,7 \\
\hline
\end{tabular}

: Categoria de referência

t: Inscrição em lista de espera foi realizada em 2018 ou em 2019

*: Por forma a garantir a não identificação dos hospitais omitiram-se estas estatísticas. Há três hospitais de origem de maior dimensão que perfazem $79 \%$ da amostra.

Relativamente a questões éticas, foi solicitada à ARSC, IP autorização para usar os dados com vista a submeter um artigo a uma revista científica. O pedido foi reencaminhado para o Ministério da Saúde. Na sequência do Despacho N. ${ }^{\circ}$ $6741 / 2019$ de 29 de julho, ${ }^{24}$ a autorização foi concedida, tendo sido rececionada por uma das autoras, via email, no dia 23 de agosto de 2019. Na solicitação supramencionada, foi manifestado o compromisso de não revelar a identificação dos hospitais. Consequentemente, os hospitais estão identificados apenas com números e suprimiram-se as estatísticas descritivas que de algum modo poderiam permitir a sua identificação.

\section{RESULTADOS}

A percentagem de recusas foi de 31 (Tabela 1). Existiu uma maior proporção de utentes do sexo feminino (57\%) e a idade mais prevalente corresponde à faixa 65 - 74 anos com $25 \%$ da amostra, seguida da faixa 75 - 84 anos com $23 \%$. Existiu equilíbrio entre o número de utentes à espera de cirurgia em ambulatório e o número à espera de cirurgia convencional e Oftalmologia é a especialidade que sobressai na amostra com uma prevalência de $33 \%$. Apenas $8 \%$ dos utentes em lista de espera configuraram uma situação prioritária ou muito prioritária. Em termos de hospitais de origem, há três hospitais que conjuntamente foram responsáveis por $79 \%$ das inscrições em LIC. Por fim, a esmagadora maioria dos utentes inscritos, apresentaram 2018 ou 2019 como ano de inclusão na lista.

Em relação à evolução trimestral do número de NT/ VC emitidos e recusados, de acordo com a Fig. 1, com exceção do primeiro trimestre de 2018, o número de emissões foi sempre superior após a implementação dos novos TMRG comparado com o período anterior a estes novos TMRG. Entre o segundo trimestre de 2018 e o segundo trimestre de 2019, as NT/VC emitidos ultrapassaram as 12 000 unidades, enquanto o valor mais alto atingido antes da redefinição do TMRG foi de 9386 no quarto trimestre de 2016. Em relação à proporção de recusas, o valor mais baixo no período em análise foi registado no primeiro trimestre de 2017 (30\%) e o valor mais elevado no quarto trimestre de 2019 (68\%). Em geral, as proporções de recusas foram superiores no período após a implementação dos novos TMRG comparado com o período anterior. A única exceção corresponde aos $51 \%$ do segundo trimestre de 2018 , que ficou abaixo dos 55\% do quarto trimestre de 2017. Todavia, é de sublinhar que, com exceção do quarto trimestre de 2016 (que foi atípico no período anterior a 2018 - no âmbito de um Relatório do Tribunal de Contas, a ACSS relatou constrangimentos no processo de impressão de NT/VC em $2016,{ }^{22}$ e assim, este valor atípico no último trimestre poderá dever-se à recuperação de atrasos nos trimestres anteriores), a proporção de recusas vinha a aumentar de modo muito marcante mesmo antes dos novos TMRG. Entre o primeiro trimestre de 2017 e o quarto trimestre de 2017 observou-se um aumento em todos os períodos, passando-se de $30 \%$ para $55 \%$ de recusas; após o primeiro trimestre de 2018 , as percentagens de recusas alcançaram uma estabilização relativa embora a níveis elevados (sensivelmente entre $55 \%-65 \%$ ). De facto, nos três primeiros trimestres de 2019 a percentagem de recusas ficou aquém dos $60 \%$, sugerindo uma redução face a 2018. Contudo, no último trimestre de 2019, assistiu-se a duas alterações significativas face à evolução anterior - o número de emissões diminuiu 


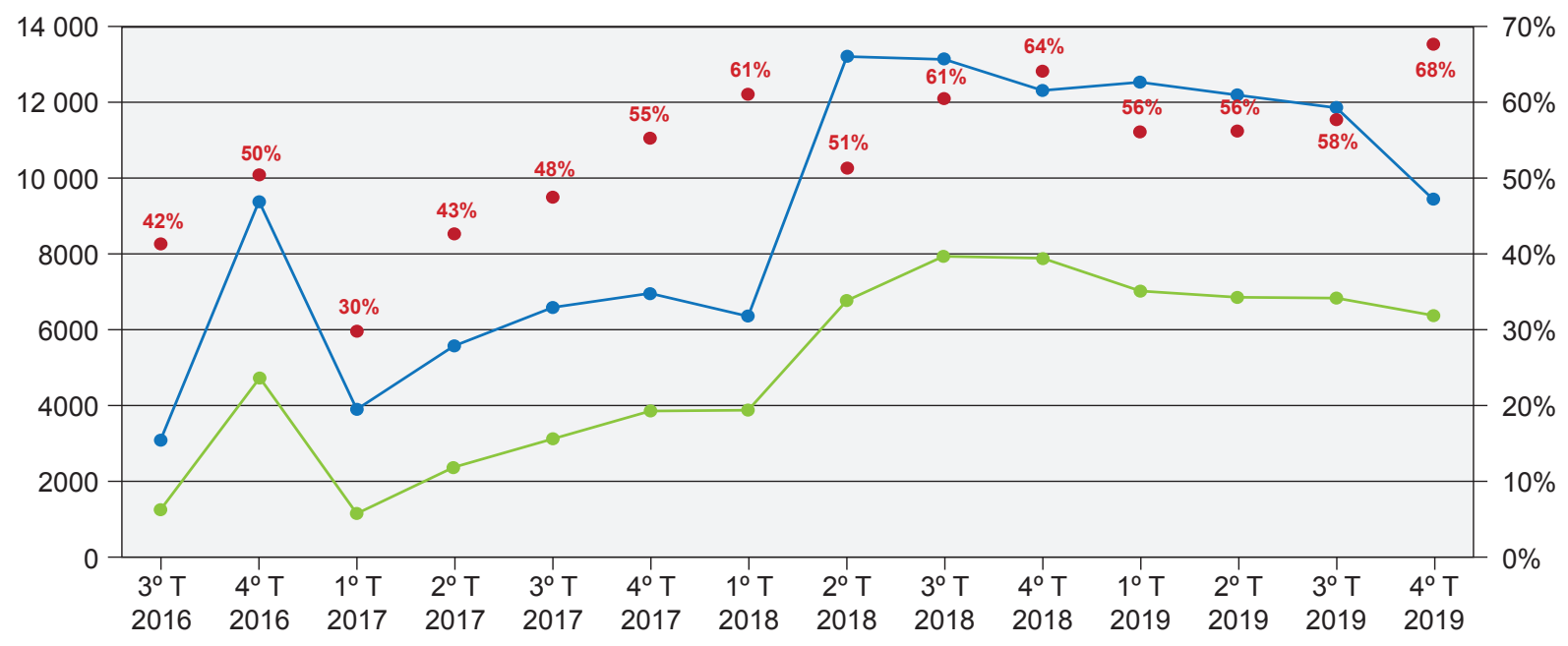

$\longrightarrow$ NT/VC emitidos (n) $\quad \longrightarrow$ NT/VC cancelados por recusa $(n) \quad \bullet$ NT/VC cancelados por recusa

Figura 1 - Notas de transferência (NT) e vales cirurgia (VC) emitidos e cancelados por recusa de transferência entre 1 de julho de 2016 e 31 de dezembro de 2019 na Região Centro

em um quinto enquanto a percentagem de recusas foi no sentido inverso e aumentou 10 pontos percentuais (o que representa no caso em questão um aumento de 17\%).

Em relação à análise de regressão, o teste Omnibus mostrou que a diferença entre o modelo apenas com a constante e o modelo adicionado das variáveis explicativas é estatisticamente significativa $(p<0,001)$. Os resultados para a razão de chances encontram-se na Tabela 2.

Os resultados não evidenciaram uma associação entre o sexo do utente e a recusa de NT/VC mas esta aumentou com a idade - para a faixa etária 55 - 64 a chance de recusa foi 1,14 vezes superior e para as faixas etárias 65 - 74 e 75 - 84 esta chance foi 1,10 vezes superior, todas em comparação com a faixa etária dos mais novos (18 - 44 anos). Em relação aos hospitais de origem, comparados com o HO_1, em três hospitais a chance de recusa foi inferior e nos restantes foi superior. No caso de se tratar de uma cirurgia classificada com nível superior de prioridade, a chance de recusa foi menor face à prioridade normal. Os utentes à espera de cirurgia convencional apresentaram uma chance de recusa 2,5 vezes superior à dos utentes à espera de cirurgia em ambulatório. Os utentes na especialidade de Oftalmologia correspondem ao grupo com maior peso e apresentaram uma chance de recusa inferior face aos utentes na Cirurgia Geral. Já para a especialidade de Ortopedia os resultados sugeriram uma chance de recusa superior por comparação com Cirurgia Geral. Por fim, a chance de recusa foi duas vezes inferior entre os utentes inscritos em 2018 ou depois em comparação com quem foi inscrito antes de 2018.

\section{DISCUSSÃO}

Os TMRG constituem um mecanismo utilizado em Portugal, como em vários países, com vista a reduzir os tempos de espera para cirurgia. Quando são atingidos os $50 \%$ do TMRG é emitida uma NT e aos $75 \%$ do tempo é emitido um VC. Contudo, a taxa de utilização das NT/VC tem sido muito baixa (na ordem dos $20 \%$ no triénio 2014 - 2016). ${ }^{22}$ Note-se que neste estudo analisámos os cancelamentos por recusa de transferência, mas a este motivo acrescem outros (por exemplo, desistência, já operado e erro administrativo). Assim, o total de cancelamentos é superior aos apresentados nos nossos resultados. Os TMRG foram redefinidos em 2017, com efeitos a partir de 1 de janeiro de 2018. Um dos objetivos deste trabalho foi analisar a evolução dos NT/VC emitidos e recusados desde 2016 até 2019, por forma a perceber se se perceciona uma mudança de comportamento após 2018. Pelos nossos resultados, houve um aumento notório na emissão de NT/VC após 2018 e uma relativa estabilização da proporção de recusas a níveis mais elevados face ao período anterior a 2018. Em todo o caso, registava-se uma tendência de subida da percentagem de recusas antes mesmo dos novos TMRG.

Em julho de 2018, o grupo técnico independente (GTI), constituído para avaliar os sistemas de gestão do acesso a cuidados no SNS, alertava para o facto da redução legal dos TMRG, sem o aumento da capacidade de resposta das instituições do SNS, acarretar o aumento das emissões de NT/VC mas com pouco impacto na redução de listas de espera devido à baixa taxa de utilização. ${ }^{22}$ Os nossos resultados confirmam este aumento das emissões e a persistência de taxas de recusas muito elevadas. O relatório do GTI aponta algumas lacunas a todo o processo das NT/ VC, tais como a carência de informação transparente sobre a qualidade dos resultados de desempenho das unidades hospitalares (não chega saber os tempos de espera) e os problemas de informação por parte dos prestadores ou das entidades responsáveis aos doentes/utentes, incluindo a informação sobre NT/VC. O problema da informação foi identificado logo nos anos iniciais do SIGIC. Um estudo com 570 entrevistas telefónicas, ${ }^{21}$ sobre os motivos de recusa no primeiro semestre de 2007, apurou que uma das 
Tabela 2 - Análise da associação entre fatores de risco e recusa de NT/VC

\begin{tabular}{|c|c|c|}
\hline Variável & $\begin{array}{c}\text { Razão de } \\
\text { chances }\end{array}$ & $\begin{array}{c}\text { Intervalo } \\
\text { confiança } \\
(95 \%)\end{array}$ \\
\hline \multicolumn{3}{|l|}{ Sexo } \\
\hline Masculino & 1,017 & 0,$966 ; 1,071$ \\
\hline \multicolumn{3}{|l|}{ Idade } \\
\hline Idade_45_54 & 1,036 & 0,$941 ; 1,140$ \\
\hline Idade_55_64 & $1,136^{\text {**** }}$ & 1,$041 ; 1,240$ \\
\hline Idade_65_74 & $1,095^{* *}$ & 1,$005 ; 1,194$ \\
\hline Idade_75_84 & $1,098^{* *}$ & 1,$002 ; 1,203$ \\
\hline Idade_85+ & 1,020 & 0,$891 ; 1,168$ \\
\hline \multicolumn{3}{|l|}{ Hospital de origem } \\
\hline HO_2 & $3,853^{\text {*t*k}}$ & 3,$610 ; 4,113$ \\
\hline HO_3 & $0,654^{* * *}$ & 0,$573 ; 0,746$ \\
\hline $\mathrm{HO}_{-} 4$ & $2,751^{* * *}$ & 3,$383 ; 3,175$ \\
\hline HO_5 & $0,706^{* * *}$ & 0,$583 ; 0,853$ \\
\hline HO_6 & $1,337^{* * *}$ & 1,$092 ; 1,637$ \\
\hline HO_7 & $3,600^{* * *+}$ & 3,$171 ; 4,087$ \\
\hline HO_8 & 0,972 & 0,$807 ; 1,171$ \\
\hline HO_9 & $1,371^{\text {**t* }}$ & 1,$266 ; 1,486$ \\
\hline HO_outros & $0,007^{*+*}$ & 0,$002 ; 0,027$ \\
\hline
\end{tabular}

Nível de prioridade da cirurgia

$\begin{array}{ccc}\begin{array}{c}\text { Prioritário_MP } \\ \text { Modalidade da cirurgia }\end{array} & 0,638^{*+*+*} & 0,577 ; 0,706 \\ \text { Cir_convenc } & 2,498^{*+*+*} & 2,343 ; 2,663\end{array}$

Especialidade da cirurgia

$\begin{array}{lll}\text { Oftalmologia } & 0,783^{*+*} & 0,714 ; 0,857 \\ \text { Ortopedia } & 1,123^{*+*} & 1,037 ; 1,217 \\ \text { Outras_esp } & 0,732^{*+*} & 0,675 ; 0,794\end{array}$

Período posterior/anterior à redefinição dos TMRG

Ano_inclusão $\quad 0,447^{*+*} \quad 0,400 ; 0,500$

Nota - categorias de referência: sexo feminino; idade_18_44; HO_1; Prioridade normal; Cirurgia de ambulatório; Cirurgia geral; inscrição em lista de espera anterior a 2018. ${ }^{* * *}: p<0,01 ; * *: p<0,05 ; *: p<0,1$

$R^{2}$ Nagelkerke $=0,206$

razões de recusa era precisamente o défice de informação (embora tenha sido o motivo com menor expressão). À data em que escrevemos este artigo, as NT/VC foram, entretanto, alvo de um redesenho, com informação melhorada para apoiar o utente na sua decisão. Estudos futuros poderão averiguar o seu impacto sobre a taxa de recusas.

No contexto de uma tão baixa utilização de NT/VC é importante investigar os fatores de risco associados à recusa e este é outro objetivo do presente estudo. Pelos nossos resultados, eventuais medidas políticas não necessitam de discriminar os utentes por sexo uma vez que não se encontraram diferenças a este nível. Os mais novos também parecem ser menos propensos à recusa, e portanto os esforços para melhorar a utilização de NT/VC devem centrar-se nos utentes com idades superiores a 55 anos.
Merecem particular atenção os utentes à espera para cirurgia convencional e na especialidade de Ortopedia, onde a chance de recusa parece ser superior. Em termos de unidades hospitalares, os resultados sugerem a existência de algumas diferenças, em alguns casos, bastante acentuadas. O compromisso da não identificação dos hospitais não nos permite, no entanto, formular recomendações a este respeito.

Pela natureza dos dados usados, não é possível apurar as razões que levaram à recusa. $O$ único estudo sobre esse tema utilizou dados de há mais de 10 anos. ${ }^{21} \mathrm{~A}$ principal razão para a recusa de transferência foi a preferência pela equipa médica e hospital com quem o utente já se sente familiarizado, seguido da indisponibilidade para usar a NT/ VC dentro do prazo de validade e a relutância em mudar de área de residência. $\mathrm{O}$ cruzamento desta informação com as características dos utentes/cirurgias identificados no nosso estudo poderá ser útil no desenho de medidas para aumentar a eficácia das NT/VC, sob pena de estar a ser realizada despesa com todo este processo sem retorno.

\section{Limitações}

O presente estudo vem preencher uma lacuna na literatura, dada a quase total ausência de análises sobre a baixa utilização de NT/VC. Dois pontos fortes do nosso trabalho prendem-se com o uso de dados administrativos e de um elevado número de observações. No entanto, existem algumas limitações. Assumiu-se que para tempos de espera para cirurgia iguais ou superiores a $50 \%$ houve emissão de NT/VC. Tal poderá não ser o caso, pelo que a nossa amostra pode incluir episódios em que a variável 'Recusa' toma o valor zero, quando efetivamente essas observações deveriam ser eliminadas. Por outro lado, a base de dados de NT/VC cancelados inclui recusas ocorridas no máximo até 31 de dezembro de 2019. Consequentemente, é provável que alguns utentes à espera de cirurgia a 31 de dezembro tenham recusado as respetivas NT/VC após esta data e, como tal, não estão contemplados no nosso estudo. Estas duas razões poderão explicar a discrepância entre a taxa de recusas que emergiu da análise de regressão e as taxas na Fig.1. A informação em relação à associação entre a recusa e o hospital de origem é limitada pela impossibilidade de revelar a identificação dos hospitais. Contudo, a análise foi desenvolvida e consideramos ser em todo o caso relevante alertar para este achado. Os nossos resultados sugerem que a chance de recusa foi menor para inscrições em LIC a partir de 2018, apesar da esmagadora maioria dos utentes em espera a 31 de dezembro terem sido inscritos após 2018 e os inscritos anteriormente poderão ter atingido $50 \%$ do TMRG já em 2018. Assim, este resultado deve ser lido com cautela. Os nossos dados aplicam-se apenas à Região Centro, pelo que outros estudos serão necessários em relação às restantes regiões do país. Os resultados da análise de regressão mostraram que a diferença entre o modelo usado e o modelo apenas com a constante é estatisticamente significativa. Contudo, a percentagem de observações corretamente previstas foi, no total, de $71,3 \%$, 
tendo sido consideravelmente mais baixa nos casos de recusa. Por outro lado, não foi possível obter através das fontes de dados utilizadas neste estudo informação relativa às alternativas dos hospitais de destino oferecidas ao utente. Esta informação é potencialmente relevante para melhor investigar a decisão de recusa, nomeadamente, no que tem que a ver com a distância entre os hospitais de destino e o de origem, um fator já identificado na literatura como tendo impacto na escolha do hospital. ${ }^{16}$ Efetivamente, pela natureza dos dados utilizados, a nossa análise restringiu-se ao impacto de fatores administrativos e de gestão, tendo elementos como a confiança na equipa médica e amenidades da entidade escolhida ficado de fora. Apesar destas limitações, é nossa convicção que os resultados alcançados são pertinentes e poderão informar a política de saúde sobre um problema que persiste. Chama-se ainda a atenção que, caso os utentes não sejam inscritos em LIC na data da consulta em que foi verificada a necessidade de cirurgia, ${ }^{22}$ os tempos de espera, mesmo com base em dados administrativos, poderão estar subestimados.

\section{CONCLUSÃO}

Os tempos de espera para cirurgia continuam a ser uma preocupação de política em Portugal e nos países da OCDE, a qual deverá agravar-se com os cancelamentos devido à pandemia de COVID-19. Neste estudo confirmou-se que a emissão de NT/VC aumentou após a redução legal dos TMRG em 2018 e que as taxas de recusa de transferência vinham já a registar uma tendência de aumento desde 2016 , tendo-se mantido acima dos 55\% a partir do terceiro trimestre de 2018. Alguns fatores para os quais se encontrou uma associação positiva com a recusa são a idade, a cirurgia convencional (em comparação com ambula- tório) e a especialidade de Ortopedia (em comparação com Cirurgia Geral). São necessários mais estudos em outras regiões do país que também analisem os motivos de recusa de transferência, de modo a desenhar estratégias para aumentar a taxa de utilização das NT/NC e reduzir o atual desperdício de recursos com a emissão de milhares de NT/ VC que vêm posteriormente a ser cancelados.

\section{CONTRIBUTO DOS AUTORES}

SC, CQ, PA: Conceção do estudo, análise/interpretação de dados, elaboração do manuscrito e aprovação da versão submetida.

\section{PROTECÇÃO DE PESSOAS E ANIMAIS}

Os autores declaram que os procedimentos seguidos estavam de acordo com os regulamentos estabelecidos pelos responsáveis da Comissão de Investigação Clínica e Ética e de acordo com a Declaração de Helsínquia da Associação Médica Mundial revista em 2013.

\section{CONFIDENCIALIDADE DOS DADOS}

Os autores declaram ter seguido os protocolos do seu centro de trabalho acerca da publicação de dados.

\section{CONFLITOS DE INTERESSE}

Os autores declaram não ter conflitos de interesse relacionados com o presente trabalho.

\section{FONTES DE FINANCIAMENTO}

O Centre for Business and Economics Research é financiado pela Fundação para a Ciência e Tecnologia, I.P., Projeto UIDB/05037/2020.

\section{REFERÊNCIAS}

1. Siciliani L, Borowitz M, Moran V, editores. Waiting time policies in the health sector: what works? OECD Health Policy Studies. Paris: OECD Publishing; 2013.

2. Organisation for Economic Co-operation and Development. Waiting times for health services: next in line. OECD Health Policy Studies. Paris: OECD Publishing; 2020

3. Siciliani L, Hurst J. Explaining waiting times variations for elective surgery across OECD countries. OECD Econ Studies. 2004;38:95-123.

4. Dixon $\mathrm{H}$, Siciliani L. Waiting-time targets in the healthcare sector. How long are we waiting?. J Health Econ. 2009;28:1081-98.

5. Goddard J, Malek M, Tavakoli M. An economic model of the market for hospital treatment for non-urgent conditions. Health Econ. 1995;4:41-5.

6. Iversen T. A theory of hospital waiting lists. J Health Econ. 1993;12:5571.

7. Guttmann A, Schull MJ, Vermeulen MJ, Stukel TA. Association between waiting times and short-term mortality and hospital admission after departure from emergency department: population-based cohort study from Ontario, Canada. BMJ. 2011;342:d2983.

8. Sobolev BG, Fradet G, Kuramoto L, Rogula B. An observational study to evaluate 2 target times for elective coronary bypass surgery. Med Care. 2012;50:611-19.

9. Nikolova S, Sinko A, Sutton M. Do maximum waiting times guarantees change clinical priorities for elective treatment? Evidence from Scotland. J Health Econ. 2015;41:72-88.

10. Siciliani L, Verzulli R. Waiting times and socioeconomic status among elderly Europeans: evidence from SHARE. Health Econ. 2009;18:1295306.

11. Laudicella M, Siciliani L, Cookson R. Waiting times and socioeconomic

status: evidence from England. Soc Sci Med. 2012;74:1331-41.

12. Entidade Reguladora da Saúde. Gestão da lista de inscritos para Cirurgia no SNS. Porto: ERS; 2014.

13. Dawson D, Gravelle H, Jacobs R, Martin S, Smith P. The effects of expanding patient choice of provider on waiting times: evidence from a policy experiment. Health Econ. 2007;16:113-28.

14. Gutacker N, Siciliani L, Moscelli G, Gravelle H. Choice of hospital: which type of quality matters? J Health Econ. 2016;50:230-46.

15. Moscelli G, Siciliani L, Gutacker N, Gravelle H. Location, quality and choice of hospital: evidence from England 2002-2013. Reg Sci Urban Econ. 2016;60:112-24.

16. Mosadeghrad AM. Patient choice of a hospital: implications for health policy and management. Int J Health C Qual Ass. 2014;27:152-64.

17. Antunes P, Ferreira PL. Gestão integrada do acesso. In: Observatório Português dos Sistemas de Saúde, editor. Meio caminho andado Relatório de Primavera. 2018. p. 153-8.

18. Portugal. Lei n. ${ }^{\circ}$ 41/2007. Diário da República, I Série, $n^{\circ} 163$ (2007/08/24). p.5664-5.

19. Portugal. Portaria $n .^{\circ} 1529 / 2008$. Diário da República, I Série, $n^{\circ} 249$ (2008/12/26). p.9040-4.

20. Portugal. Portaria n. ${ }^{\circ} 45 / 2008$. Diário da República, I Série, $n^{\circ} 10$ (2008/01/15). p.526-36.

21. Unidade Central de Gestão de Inscritos para Cirurgia. Estudo dos motivos de recusa de transferência: inquérito telefónico. Lisboa: UCGIC; 2008.

22. Guimarães $M$, (coord.). Avaliação dos sistemas de gestão do acesso a cuidados de saúde no Serviço Nacional de Saúde - Relatório. 2018. Grupo Técnico Independente. [consultado 2019 set 18]. Disponível em: 
https://www.sns.gov.pt/wp-content/uploads/2019/04/GTI.pdf.

23. Portugal. Portaria n.ำ 153/2017. Diário da República, I Série, nº 86 (2017/05/04). p.2204-9
24. Portugal. Despacho n. ${ }^{\circ} 6741 / 2019$. Diário da República, II Série, nº 143 (2019/07/29). p.77. 